\title{
Morphology, Blood Chemistry and Behavioral Response of Crossbred Chickens Reared Under Alternative Production Systems
}

Original Article

-Author(s)

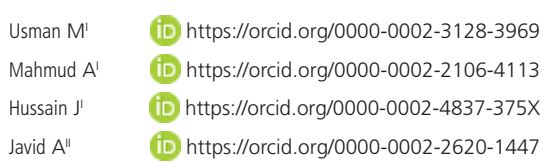

Department of Poultry Production, Faculty of Animal Production and Technology, University of Veterinary and Animal Sciences, Lahore, 54000, Pakistan.

" Department of Wildlife and Ecology, Faculty of Fisheries and Wildlife, University of Veterinary and Animal Sciences, Lahore, 54000, Pakistan.

\section{-Mail Address}

Corresponding author e-mail address Muhammad Usman

Department of Poultry Production, Faculty of Animal Production and Technology,

University of Veterinary and Animal

Sciences, Lahore-Pakistan.

Phone: +92-322-8024228

Email: musman@uvas.edu.pk

\section{- Keywords}

Alternative production systems; antibody response; genotypes; morphometrics; serum chemistry.

\section{ABSTRACT}

In the present study, the second generation of two genotypes RNN (Rhode Island Red $\times$ Naked Neck) and BNN (Black Australorp $\times$ Naked Neck) obtained by two self-crosses (RNN $\times$ RNN $=R R$ and $B N N \times B N N=$ $\mathrm{BB})$ and two reciprocal crosses (RNN $\times \mathrm{BNN}=\mathrm{RB}$ and $\mathrm{BNN} \times \mathrm{RNN}=\mathrm{BR})$ were evaluated in three alternative production systems (conventional cages, enriched cages, and aviary). In total 480 birds, comprising 240 pullets with 240 cockerels (60 pullets \& 60 cockerels from each crossbred) were used during rearing phase (17-21 weeks). Higher body weight, shank circumference, body and drumstick length were noticed in RB and BR genotypes. Among different production systems, chickens reared in enriched cages showed higher body weight and body lengths than other production systems. Comparing the behavior of chickens, higher feeding, jumping and dust bathing were observed in chickens reared in aviary systems than in other production systems while the maximum perching behavior was showed by the chickens reared in enriched cages. The highest glucose level was observed in RR genotypes when reared in aviary systems. Antibody titers against ND and IB were highest in chickens reared in aviary systems. In conclusion, RB and BR genotypes had better performance in terms of morphometrics and blood biochemistry when reared under enriched cages and aviary systems.

\section{INTRODUCTION}

The increasing world population is tremendously demanding food production and measures to increase animal protein sources. In many countries, different strategies have been applied to develop a dualpurpose rural chicken breed with further improvement in its production traits (Mallia, 1999). The genetic improvement can be achieved through crossbreeding and selection. However, cross breeding is better in terms to instigate improvements in growth rate, reproductive traits and feed conversion efficiency without disturbing potential of acclimatization ultimately reducing production costs (Adebambo et al., 2011). Better productive performance and adaptability traits of naked neck can be exploited through heterosis led by its crossbreeding with Rhode Island Red and Black Australorp which have genes of better egg production and meat yield with higher potential of economic returns helping to develop a crossbred with improved production potential and maintained acclimatizing abilities.

In association with the genetic improvement, better management and provision of suitable environment is necessary for the exploitation of genetic potential (Menge et al., 2005). Traditional conventional cages have been long perceived as the most proficient and efficient system for egg type chicken but the negative effect of cage system 
on welfare of chicken is also being considered widely (Craig \& Swanson, 1994). It has been observed that confinement and complexity in conventional cages restricts the birds to limited natural habitat which eliminates the expression of natural behaviors like roosting, nesting and scratching (Baxter, 1994). Banning of conventional cages by European Union and to overcome the lack of natural behaviors, the alternative housing systems are gaining strength day by day to cope with the consumer's demand. It is assumed that alternative systems can help the birds not only to express their natural behavior but also enhance the economical production traits.

Blood biochemistry is an ideal evaluator of health profile and is frequently used to assess immune status by avian pathologists. Among different parameters of blood biochemistry, serum proteins are useful in estimating the quality of dietary proteins (Alikwe et al., 2010). Similarly, glucose and triglycerides are useful in drawing inference about energy requirement for the body's biochemical functions and physiological response (Kral \& Suchy, 2000). Information regarding the immune status is mandatory to understand the level of disease resistance against infectious diseases. Indigenous chickens can be used as the most efficient model to study immune status against different viral and bacterial infections (Haunshi et al., 2011). The availability of data regarding the performance of different crossbreds of Naked Neck, Black Australorp and Rhode Island Red under aviary and enriched cages is limited and need to be explored. So, its need of the time to work on developing new genotypes with improved growth, better immune response and better acclimatization to local climatic condition and to evaluate their genetic potential under alternative production systems.

\section{MATERIALS AND METHODS}

The present project was planned to evaluate the performance of different crossbreds of Naked Neck (NN), Black Australorp (BAL) and Rhode Island Red (RIR) under alternative production systems i.e., Conventional Cages, Aviary systems and Enriched Cages. The study was conducted at the Indigenous Chicken Genetic resource Centre (ICGRC), Department of Poultry Production, UVAS, Ravi Campus Pattoki, Pakistan. Pattoki city is located at $73^{\circ} 50^{\prime} 60 \mathrm{E}$ and $31^{\circ} 1^{\prime} \mathrm{ON}$ with the altitude of $610 \mathrm{ft}(186 \mathrm{~m})$ and normally experiences tropical hot and humid climate where the temperature ranges between $12^{\circ} \mathrm{C}$ to $45^{\circ} \mathrm{C}$.

\section{Population Size}

The present study was in the continuation of the previous research project in which the performance of progeny (F1) taken from Rhode Island Red $\times$ Naked Neck (RNN) and Black Australorp $\times$ Naked Neck (BNN) was evaluated (Ahmad et al., 2019). In the present study, the second generation (F2) of these crossbreds (BNN and RNN) were evaluated in reciprocal crosses. For this purpose, a total of 200 heterozygous partial feather chickens (discarding homozygous full feathered and homozygous naked neck) comprising 50 birds (10 $\square \times 40 \square$ ) from each crossbred of first generation i.e.; Crossbred I (BNN $\square \times$ BNN $\square$ ), Crossbred II (BNN $\square \times$ RNN $\square$ ), Crossbred III (RNN $\square \times$ RNN $\square$ ) and Crossbred IV (RNN $\square \times$ BNN $\square$ ) were used to take more than 1200 hatching eggs in the 33rd week of age (Figure 1). The birds were arranged according to a Completely Randomized Design (CRD). the birds were fed commercial breeder ration formulated according to the recommendations of Leeson \& Summer (2005) (Table 1, 2). The chicks were brooded

Table 1 - Specification for each production system.

\begin{tabular}{|c|c|c|c|}
\hline Specifications & $\begin{array}{l}\text { Conventional cage } \\
\text { (Separate for each sex) }\end{array}$ & $\begin{array}{l}\text { Enriched cage } \\
\text { (Separate for each sex) }\end{array}$ & $\begin{array}{c}\text { Aviary } \\
\text { (Straight run) }\end{array}$ \\
\hline Dimensions (Length $\times$ Depth $\times$ Height) $(\mathrm{ft})$ & $2 \times 2.5 \times 2$ & $3 \times 3 \times 3$ & $10 \times 10 \times 10$ \\
\hline Stocking Density & 5 birds/ cage & 5 birds/cage & 50 birds / aviary \\
\hline Floor space per bird & $1.08 \mathrm{ft}^{2}\left(931.9 \mathrm{~cm}^{2}\right)$ & $1.75 \mathrm{ft}^{2}\left(1672 \mathrm{~cm}^{2}\right)$ & $2 \mathrm{ft}^{2}\left(1858 \mathrm{~cm}^{2}\right)$ \\
\hline $\begin{array}{l}\text { Dimensions of Dust bathing Area (Length } \times \text { width) } \\
(\mathrm{ft})\end{array}$ & ------------------------ & $1.79 \times 0.83$ & $4.5 \times 4.5$ \\
\hline Dust bathing Space/bird & ------------------------- & $0.3 \mathrm{ft}^{2}\left(276 \mathrm{~cm}^{2}\right)$ & $0.4 \mathrm{ft}^{2}\left(376 \mathrm{~cm}^{2}\right)$ \\
\hline \multicolumn{4}{|l|}{ Perches } \\
\hline Number & 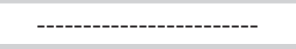 & 1 & 2 \\
\hline Material & ------------------------ & Wooden & Wooden \\
\hline Shape & ------------------------- & Round & Round \\
\hline Diameter $(\mathrm{cm})$ & 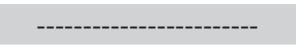 & 1.8 & 4.2 \\
\hline Length & & $4 \mathrm{ft}$ & $10 \mathrm{ft}$ \\
\hline Height from floor & & $1.5 \mathrm{ft}$ & $3 \mathrm{ft}$ \\
\hline
\end{tabular}


Table 2 - Weekly feed allowance (g) during growing phase.

\begin{tabular}{lc}
\hline Age (weeks) & Daily feed allowance $(\mathrm{g})$ \\
\hline 17 & 44 \\
18 & 46 \\
19 & 48 \\
20 & 50 \\
21 & 52 \\
\hline
\end{tabular}

under standard managemental conditions up to 6 weeks of age. During brooding phase, the birds were vaccinated against IB and ND according to the local area schedule. After brooding, the birds were divided into three different production systems (conventional cages, enriched cages and aviary systems). A total of 480 birds comprising of 240 pullets with 240 cockerels ( 60 pullets and 60 cockerels from each crossbred) were used to evaluate the performance during rearing phase (17-21 weeks).

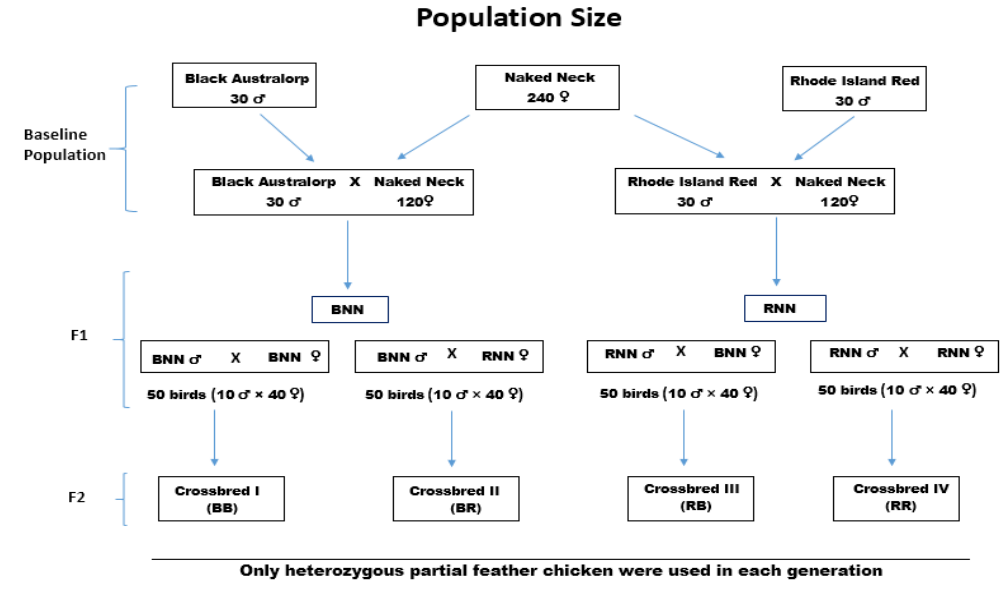

Figure 1 - Population size.

\section{Production systems}

Chickens reared in enriched cages and aviary systems were provided with perches and dust bathing area. Open sided windowed enclosures ventilated with ceiling fans were used for aviaries and enriched cages.
Galvanized round feeders and plastic manual drinkers were used in aviaries and enriched cages. The physical characteristics of each system is explained in Table (3). Birds reared in conventional cage systems were maintained in environmental controlled poultry shed equipped with galvanized three-tiered battery cage system, automatic manure belt, automatic water nipple line and feed trolley (FACCO, Poultry Equipment-C3). Supply of ad libitum fresh water was ensured.

\section{Housing conditions}

The duration of the experiments was 5 weeks (June and July) during which the minimum to maximum temperature and humidity were maintained between the range of $28^{\circ} \mathrm{C}$ to $35^{\circ} \mathrm{C}$ and $62 \%$ to $77 \%$ respectively inside open sided enclosures while in environmentally control houses the minimum and maximum temperature and humidity ranged between $26^{\circ} \mathrm{C}$ to $32^{\circ} \mathrm{C}$ and $66 \%$ to $74 \%$ respectively. Rice husk was used as litter material in both enriched cages and aviaries. Approximately 4 inches depth of bedding material was maintained and racking on daily basis was ensured to uphold its better condition.

\section{Parameters evaluated}

Data were collected for morphometric measurements on a weekly basis during rearing phase from 17- 21 weeks in terms of body, keel, drumstick and shank length, wing spread and drumstick and shank circumference with the help of measuring tape. To evaluate serum chemistry, $3 \mathrm{ml}$ of blood collected from the brachial vein of 3 birds per treatment group was centrifuged to collect serum in Eppendorf tubes and stored at $-20^{\circ} \mathrm{C}$ (Gunes et al., 2002). The serum was analyzed by the method adopted by Kumar \& Kumbhakar (2015). Antibody response against NDV was evaluated by Hemagglutination inhibition technique (HI) (Xie et al., 2008).

Table 3 - Nutrition value and composition of experimental ration during growing phase.

\begin{tabular}{lccc}
\hline Feed Ingredient (\%) & Grower (17-21 weeks) & Nutritive value & Grower (17-21 weeks) \\
\hline Corn & 59 & CP (\%) & 15.46 \\
Soybean Meal & 7 & ME (Kcal/Kg) & 2913 \\
Rice tips & 8.4 & Ca (\%) & 1 \\
Soybean Oil & 1.2 & Av. P (\%) & 0.42 \\
Wheat grain & 5 & Lysine (\%) & 0.69 \\
Wheat bran & 5 & Methionine (\%) & 0.39 \\
Canola Meal & 10 & & \\
Limestone & 2.4 & & \\
Feather Meal & 1.1 & & \\
NaCl & 0.30 & & \\
Methionine & 0.12 & & \\
Total & 100 & & \\
\hline
\end{tabular}




\section{Behavior}

To evaluate the behavioral and welfare related traits, the birds from each treatment groups were observed keenly, 11:00 AM to 01:00 PM on a weekly basis. Welfare traits were included dust bathing, jumping, perching, sitting and walking (Costa et al., 2012).

\section{Statistical Analysis}

The effect of production systems and genotypes were evaluated on morphometric measurements and carcass traits. Two-way ANOVA technique was used for analysis assuming production systems and genotypes as main effects. Tukey's HSD test (Tukey, 1953) was employed to compare treatment means at significance level of $p \leq 0.05$. Mathematical model is given.

$$
Y_{i j k}=\mu+P_{i}+G_{j}+(P \times G)_{i j}+\varepsilon_{i j k}
$$

Where,

$Y_{i j k}=$ Observation of dependent variable recorded on $i^{\text {th }}$ Production System and $j^{\text {th }}$ Genotype

$$
\begin{aligned}
& \mu=\text { Population mean } \\
& P_{i}=\text { Effect of } i^{\text {th }} \text { Production System }(i=1,2,3) \\
& G_{j}=\text { Effect of } j^{\text {th }} \text { Genotype }(j=1,2,3,4)
\end{aligned}
$$

$(\mathrm{P} \times \mathrm{G})_{\mathrm{ij}}=$ Interaction between production system and Genotype

$\varepsilon_{i j k}=$ Residual error of $k^{\text {th }}$ observation on $i^{\text {th }}$ Production system and $j^{\text {th }}$ genotype NID $\sim 0, \sigma^{2}$

\section{RESULTS}

\section{Morphometric measurements}

The effect of genotypes, production system and their interaction on body weight and morphometric measurements for males and females are explained in Tables 4 and 5. RB and BR genotype chickens (male $p<0.0001$ and female $p=0.0028$ ) were heavier than $R R$ and BB genotype chickens. Females reared in Enriched cages were heavier $(p<0.0001)$ than females reared in Aviary and Conventional cages while males from enriched cages and conventional cages showed higher $(p<0.0001)$ body weights than males reared in aviary system. The interaction between the genotypes and production systems showed the highest body weight of females $(p<0.0001)$ from BR genotype reared in Enriched cage system while males $(p<0.0001)$ of BB

\begin{tabular}{|c|c|c|c|c|c|c|c|c|c|}
\hline GT & PS & BW & $\mathrm{BL}$ & $S L$ & SC & $\mathrm{DL}$ & DC & $\mathrm{KL}$ & WS \\
\hline $\mathrm{RB}$ & & $1927.82 \pm 32.60^{a}$ & $71.32 \pm 1.80^{\mathrm{a}}$ & $10.61 \pm 0.17$ & $4.17 \pm 0.04^{a}$ & $15.18 \pm 0.23^{a}$ & $9.20 \pm 0.22$ & $13.55 \pm 0.20$ & $10.48 \pm 0.16$ \\
\hline RR & & $1787.92 \pm 37.09^{b}$ & $64.19 \pm 3.04^{\mathrm{ab}}$ & $10.08 \pm 0.18$ & $3.81 \pm 0.12^{b c}$ & $14.39 \pm 0.26^{\mathrm{ab}}$ & $8.63 \pm 0.14$ & $12.85 \pm 0.29$ & $10.25 \pm 0.20$ \\
\hline BR & & $1881.78 \pm 24.35^{a}$ & $69.34 \pm 2.05^{a}$ & $10.34 \pm 0.18$ & $4.08 \pm 0.09^{a b}$ & $14.90 \pm 0.33^{\mathrm{ab}}$ & $9.07 \pm 0.25$ & $13.09 \pm 0.27$ & $10.32 \pm 0.16$ \\
\hline BB & & $1733.26 \pm 34.02^{b}$ & $57.26 \pm 0.90^{b}$ & $10.22 \pm 0.23$ & $3.62 \pm 0.12^{c}$ & $14.06 \pm 0.21^{b}$ & $8.50 \pm 0.14$ & $12.45 \pm 0.70$ & $10.20 \pm 0.22$ \\
\hline & $C C$ & $1859.77 \pm 21.55^{a}$ & $66.55 \pm 2.38^{\mathrm{ab}}$ & $10.21 \pm 0.15$ & $4.13 \pm 0.06^{a}$ & $14.57 \pm 0.19$ & $8.80 \pm 0.21$ & $12.20 \pm 0.22^{b}$ & $10.09 \pm 0.13$ \\
\hline & AV & $1716.71 \pm 33.83^{b}$ & $61.86 \pm 1.95^{b}$ & $10.35 \pm 0.13$ & $3.72 \pm 0.10^{b}$ & $14.40 \pm 0.21$ & $8.68 \pm 0.14$ & $13.74 \pm 0.11^{\mathrm{a}}$ & $10.47 \pm 0.14$ \\
\hline & EC & $1921.61 \pm 21.56^{a}$ & $68.17 \pm 1.94^{a}$ & $10.37 \pm 0.21$ & $3.92 \pm 0.10^{\mathrm{ab}}$ & $14.93 \pm 0.30$ & $9.08 \pm 0.17$ & $13.02 \pm 0.53^{\mathrm{ab}}$ & $10.37 \pm 0.19$ \\
\hline RB & CC & $1974.89 \pm 34.05^{a}$ & $73.70 \pm 3.44^{\mathrm{ab}}$ & $10.40 \pm 0.31$ & $4.21 \pm 0.08^{\mathrm{a}}$ & $14.85 \pm 0.24$ & $9.11 \pm 0.54$ & $12.77 \pm 0.34$ & $10.24 \pm 0.34$ \\
\hline RB & AV & $1830.43 \pm 73.44^{\mathrm{ab}}$ & $72.99 \pm 3.64^{\mathrm{abc}}$ & $10.28 \pm 0.24$ & $4.16 \pm 0.07^{\mathrm{ab}}$ & $14.83 \pm 0.28$ & $8.93 \pm 0.23$ & $14.00 \pm 0.21$ & $10.44 \pm 0.18$ \\
\hline RB & EC & $1978.14 \pm 32.45^{a}$ & $67.26 \pm 1.69^{\mathrm{abcd}}$ & $11.14 \pm 0.24$ & $4.16 \pm 0.10^{\mathrm{ab}}$ & $15.87 \pm 0.48$ & $9.57 \pm 0.36$ & $13.88 \pm 0.22$ & $10.76 \pm 0.28$ \\
\hline RR & CC & $1803.58 \pm 24.46^{a b}$ & $63.81 \pm 7.33^{\mathrm{abcd}}$ & $9.72 \pm 0.20$ & $4.19 \pm 0.13^{a}$ & $14.47 \pm 0.53$ & $8.62 \pm 0.20$ & $11.94 \pm 0.63$ & $9.84 \pm 0.20$ \\
\hline RR & AV & $1638.23 \pm 42.43^{\mathrm{bc}}$ & $57.32 \pm 2.50^{d}$ & $10.54 \pm 0.40$ & $3.39 \pm 0.19^{b}$ & $14.09 \pm 0.29$ & $8.53 \pm 0.21$ & $13.35 \pm 0.07$ & $10.42 \pm 0.45$ \\
\hline RR & EC & $1921.94 \pm 43.51^{a}$ & $71.45 \pm 3.55^{\mathrm{abcd}}$ & $9.98 \pm 0.27$ & $3.85 \pm 0.14^{\mathrm{ab}}$ & $14.61 \pm 0.54$ & $8.76 \pm 0.34$ & $13.27 \pm 0.45$ & $10.48 \pm 0.36$ \\
\hline BR & CC & $1863.57 \pm 33.55^{a}$ & $70.98 \pm 1.46^{\mathrm{abcd}}$ & $10.04 \pm 0.36$ & $4.18 \pm 0.16^{a}$ & $14.96 \pm 0.36$ & $9.03 \pm 0.56$ & $12.23 \pm 0.54$ & $9.94 \pm 0.33$ \\
\hline BR & AV & $1807.20 \pm 21.26^{a b}$ & $60.30 \pm 2.66^{\text {bcd }}$ & $10.46 \pm 0.19$ & $3.81 \pm 0.14^{\mathrm{ab}}$ & $14.79 \pm 0.69$ & $8.87 \pm 0.43$ & $1378 \pm 0.31$ & $10.56 \pm 0.16$ \\
\hline$B R$ & EC & $1974.57 \pm 32.00^{a}$ & $76.74 \pm 0.65^{a}$ & $10.53 \pm 0.37$ & $4.26 \pm 0.12^{\mathrm{a}}$ & $14.96 \pm 0.73$ & $9.33 \pm 0.40$ & $13.26 \pm 0.24$ & $10.46 \pm 0.30$ \\
\hline BB & CC & $1797.01 \pm 29.22^{\mathrm{ab}}$ & $57.73 \pm 0.94^{\mathrm{cd}}$ & $10.68 \pm 0.26$ & $3.93 \pm 0.14^{\mathrm{ab}}$ & $14.00 \pm 0.36$ & $8.45 \pm 0.38$ & $11.86 \pm 0.20$ & $1035 \pm 0.15$ \\
\hline BB & AV & $1591.00 \pm 57.75^{c}$ & $56.83 \pm 1.66^{d}$ & $10.13 \pm 0.21$ & $3.53 \pm 0.25^{\mathrm{ab}}$ & $13.89 \pm 0.20$ & $8.38 \pm 0.19$ & $13.82 \pm 0.24$ & $10.46 \pm 0.36$ \\
\hline BB & EC & $1811.77 \pm 18.69^{a b}$ & $57.23 \pm 2.18^{d}$ & $9.86 \pm 0.60$ & $3.40 \pm 0.22^{b}$ & $14.27 \pm 0.55$ & $8.66 \pm 0.13$ & $11.68 \pm 2.09$ & $9.80 \pm 0.56$ \\
\hline SOV & & \multicolumn{8}{|c|}{ ANOVA } \\
\hline GT & & $<0.0001$ & $<0.0001$ & 0.2950 & 0.0004 & 0.0165 & 0.0394 & 0.2588 & 0.7490 \\
\hline PS & & $<0.0001$ & 0.0355 & 0.7681 & 0.0043 & 0.2514 & 0.2414 & 0.0085 & 0.2473 \\
\hline GT $\times$ PS & & $<0.0001$ & $<0.0001$ & 0.1820 & 0.0002 & 0.2424 & 0.4542 & 0.1484 & 0.6199 \\
\hline
\end{tabular}
genotype reared in aviary system showed the lowest body weight than other interaction groups.

Table 4 - Male morphometric measurements at 21 weeks of age.

Note: Superscripts on different means within column differ significantly at $p \leq 0.05$.

$\mathrm{GT}=$ Genotype; RB = RNN $\times$ BNN; RR = RNN $\times$ RNN; BR = BNN $\times$ RNN; BR = BNN $\times$ RNN; BB $=$ BNN $\times$ BNN; PS = Production System; CC = Conventional Cages; AV = Aviary; EC $=$ Enriched Cages; SOV = Source of Variation; BW = Body weight $(\mathrm{g}) ; \mathrm{BL}=$ Body length $(\mathrm{cm}) ; \mathrm{SL}=$ Shank length $(\mathrm{cm}) ; \mathrm{SC}=\mathrm{Shank}$ circumference $(\mathrm{cm}) ; \mathrm{DL}=\mathrm{Drumstick}$ length $(\mathrm{cm})$; $\mathrm{DC}=$ Drumstick circumference $(\mathrm{cm}) ; \mathrm{KL}=$ Keel length $(\mathrm{cm}) ; \mathrm{WS}=$ Wing spread $(\mathrm{cm}) ;$ ANOVA = Analysis of Variance. 
Table $\mathbf{5}$ - Female morphometric measurements at 21 weeks of age.

\begin{tabular}{|c|c|c|c|c|c|c|c|c|c|}
\hline GT & PS & BW & $\mathrm{BL}$ & $\mathrm{SL}$ & SC & $\mathrm{DL}$ & DC & $\mathrm{KL}$ & WS \\
\hline RB & & $1524.07 \pm 2863^{a}$ & $67.18 \pm 1.73$ & $8.90 \pm 0.56$ & $3.45 \pm 0.11$ & $14.31 \pm 0.32^{\mathrm{a}}$ & $8.62 \pm 0.23^{a}$ & $10.84 \pm 0.32$ & $10.02 \pm 0.21$ \\
\hline RR & & $1390.12 \pm 54.21^{\mathrm{ab}}$ & $62.00 \pm 3.02$ & $8.25 \pm 0.39$ & $3.29 \pm 0.18$ & $13.39 \pm 0.30^{\mathrm{ab}}$ & $7.82 \pm 0.22^{\mathrm{ab}}$ & $10.13 \pm 0.33$ & $9.53 \pm 0.21$ \\
\hline$B R$ & & $1465.09 \pm 58.87^{a}$ & $65.82 \pm 2.37$ & $8.82 \pm 0.25$ & $3.57 \pm 0.12$ & $13.98 \pm 0.46^{\mathrm{ab}}$ & $8.43 \pm 0.25^{\mathrm{ab}}$ & $11.12 \pm 0.38$ & $9.96 \pm 0.20$ \\
\hline BB & & $1317.69 \pm 36.57^{b}$ & $60.22 \pm 0.89$ & $8.03 \pm 0.40$ & $3.10 \pm 0.06$ & $12.82 \pm 0.30^{\mathrm{b}}$ & $7.63 \pm 0.31^{b}$ & $10.34 \pm 0.36$ & $9.70 \pm 0.20$ \\
\hline & CC & $1398.48 \pm 39.96^{b}$ & $64.97 \pm 2.09^{a b}$ & $8.37 \pm 0.35$ & $3.44 \pm 0.11$ & $13.70 \pm 0.29$ & $8.20 \pm 0.18$ & $10.40 \pm 0.28$ & $9.54 \pm 0.20$ \\
\hline & AV & $1319.84 \pm 35.17^{b}$ & $59.71 \pm 1.79^{b}$ & $8.74 \pm 0.38$ & $3.26 \pm 0.14$ & $13.33 \pm 0.39$ & $7.80 \pm 0.21$ & $10.74 \pm 0.26$ & $10.06 \pm 0.20$ \\
\hline & EC & $1554.41 \pm 36.76^{a}$ & $66.73 \pm 1.57^{a}$ & $8.38 \pm 0.36$ & $3.35 \pm 0.09$ & $13.85 \pm 0.28$ & $8.37 \pm 0.29$ & $10.68 \pm 0.38$ & $9.80 \pm 0.12$ \\
\hline RB & CC & $1542.62 \pm 48.47^{a b c}$ & $69.70 \pm 3.41^{\mathrm{ab}}$ & $8.88 \pm 0.81$ & $3.28 \pm 0.32^{\mathrm{ab}}$ & $14.38 \pm 0.44$ & $8.77 \pm 0.22$ & $11.14 \pm 0.67$ & $9.76 \pm 0.21^{\mathrm{ab}}$ \\
\hline RB & AV & $1499.21 \pm 32.63^{\mathrm{abc}}$ & $68.36 \pm 3.43^{\mathrm{ab}}$ & $9.75 \pm 1.13$ & $3.52 \pm 0.13^{\mathrm{ab}}$ & $13.96 \pm 0.61$ & $8.15 \pm 0.58$ & $10.40 \pm 0.44$ & $10.89 \pm 0.32^{\mathrm{a}}$ \\
\hline RB & EC & $1530.36 \pm 70.33^{\mathrm{abc}}$ & $63.48 \pm 1.68^{\mathrm{ab}}$ & $8.07 \pm 1.02$ & $3.56 \pm 0.13^{\mathrm{ab}}$ & $14.59 \pm 0.67$ & $8.95 \pm 0.30$ & $10.98 \pm 0.61$ & $9.42 \pm 0.22^{\mathrm{ab}}$ \\
\hline $\mathrm{RR}$ & CC & $1318.21 \pm 92.91^{b c}$ & $61.54 \pm 7.30^{\mathrm{ab}}$ & $7.16 \pm 0.88$ & $3.68 \pm 0.13^{a}$ & $13.52 \pm 0.31$ & $7.87 \pm 0.19$ & $10.26 \pm 0.76$ & $9.17 \pm 0.53^{b}$ \\
\hline RR & AV & $1233.21 \pm 37.28^{c}$ & $55.10 \pm 2.52^{b}$ & $9.26 \pm 0.52$ & $2.62 \pm 0.30^{b}$ & $12.93 \pm 0.46$ & $7.69 \pm 0.21$ & $9.54 \pm 0.31$ & $9.48 \pm 0.20^{\mathrm{ab}}$ \\
\hline RR & EC & $1618.95 \pm 17.40^{\mathrm{ab}}$ & $69.36 \pm 3.24^{\mathrm{ab}}$ & $8.34 \pm 0.19$ & $3.57 \pm 0.28^{\mathrm{ab}}$ & $13.71 \pm 0.76$ & $7.90 \pm 0.68$ & $10.59 \pm 0.60$ & $9.93 \pm 0.26^{\mathrm{ab}}$ \\
\hline BR & CC & $1455.47 \pm 83.32^{\mathrm{abc}}$ & $68.12 \pm 1.38^{\mathrm{ab}}$ & $7.96 \pm 0.18$ & $3.79 \pm 0.16^{a}$ & $14.05 \pm 0.67$ & $8.55 \pm 0.28$ & $10.53 \pm 0.41$ & $9.76 \pm 0.45^{\mathrm{ab}}$ \\
\hline$B R$ & AV & $1301.13 \pm 89.87^{c}$ & $55.42 \pm 3.44^{b}$ & $8.62 \pm 0.21$ & $3.76 \pm 0.26^{a}$ & $13.92 \pm 1.29$ & $7.88 \pm 0.52$ & $11.21 \pm 0.24$ & $10.28 \pm 0.38^{\mathrm{ab}}$ \\
\hline BR & EC & $1638.67 \pm 84.28^{a}$ & $73.92 \pm 0.82^{\mathrm{a}}$ & $9.88 \pm 0.39$ & $3.16 \pm 0.13^{\mathrm{ab}}$ & $13.98 \pm 0.41$ & $8.87 \pm 0.44$ & $11.61 \pm 1.06$ & $9.83 \pm 0.17^{\mathrm{ab}}$ \\
\hline BB & CC & $1277.61 \pm 33.93^{c}$ & $60.54 \pm 0.92^{\mathrm{ab}}$ & $9.49 \pm 0.34$ & $3.02 \pm 0.13^{\mathrm{ab}}$ & $12.83 \pm 0.73$ & $7.64 \pm 0.49$ & $9.66 \pm 0.22$ & $9.47 \pm 0.40^{\mathrm{ab}}$ \\
\hline BB & AV & $1245.81 \pm 39.99^{c}$ & $59.96 \pm 1.57^{\mathrm{ab}}$ & $7.36 \pm 0.65$ & $3.15 \pm 0.13^{\mathrm{ab}}$ & $12.52 \pm 0.58$ & $7.47 \pm 0.41$ & $11.82 \pm 0.45$ & $9.59 \pm 0.39^{a b}$ \\
\hline BB & EC & $1429.65 \pm 80.43^{\text {abc }}$ & $60.18 \pm 2.23^{\mathrm{ab}}$ & $7.24 \pm 0.63$ & $3.12 \pm 0.06^{\mathrm{ab}}$ & $13.11 \pm 0.22$ & $7.77 \pm 0.77$ & $9.54 \pm 0.58$ & $10.04 \pm 0.28^{\mathrm{ab}}$ \\
\hline SOV & & \multicolumn{8}{|c|}{ ANOVA } \\
\hline GT & & 0.0028 & 0.0669 & 0.4024 & 0.0770 & 0.0245 & 0.0223 & 0.2001 & 0.2986 \\
\hline PS & & $<0.0001$ & 0.0187 & 0.7185 & 0.5488 & 0.4875 & 0.1805 & 0.7051 & 0.1291 \\
\hline$G T \times P S$ & & $<0.0001$ & 0.0009 & 0.0360 & 0.0035 & 0.4785 & 0.2838 & 0.0859 & 0.0833 \\
\hline
\end{tabular}

Note: Superscripts on different means within column differ significantly at $p \leq 0.05$.

$\mathrm{GT}=$ Genotype; RB = RNN $\times$ BNN; RR = RNN $\times$ RNN; BR = BNN $\times$ RNN; BR = BNN $\times$ RNN; BB $=$ BNN $\times$ BNN; PS = Production System; CC = Conventional Cages; $A V=$ Aviary; EC $=$ Enriched Cages; SOV = Source of Variation; BW = Body weight $(\mathrm{g}) ; \mathrm{BL}=$ Body length $(\mathrm{cm}) ; \mathrm{SL}=$ Shank length $(\mathrm{cm}) ; \mathrm{SC}=\mathrm{Shank}$ circumference $(\mathrm{cm}) ; \mathrm{DL}=\mathrm{Drumstick}$ length $(\mathrm{cm})$; $D C=$ Drumstick circumference $(\mathrm{cm}) ; \mathrm{KL}=$ Keel length $(\mathrm{cm}) ; \mathrm{WS}=$ Wing spread $(\mathrm{cm}) ;$ ANOVA = Analysis of Variance

Males of RB and $B R$ genotype showed higher $(p<0.0001)$ body lengths than BB and RR genotypes while non-significant differences ( $p$ 0.0669) were observed in body lengths of females among different genotypes. Regarding production systems, chickens reared in enriched cages (male $p=0.0355$ and female $p=0.0187$ ) showed longer bodies than chickens reared in aviary and conventional cages. Chickens of BR genotypes (Males $p<0.0001$ and females $p=0.0009$ ) reared in Enriched cage systems showed significantly higher body lengths than other interaction groups.

Higher $(p=0.0004)$ shank circumference was observed in males of RB genotype followed by males of $B R, R R$ and $B B$ genotypes and conventional cage system $(p=0.0043)$ followed by Enriched cage and aviary system. Shank circumference of females did not differ significantly among the genotypes $(p=0.0770)$ and production systems $(p=0.5488)$. Significant interactions in shank circumference of both sexes (male $p=0.0002$ female $p=0.0035$ ) were noticed.

The drumstick length (male $p=0.2514$, female $p=0.4875$ ) and circumference (male $p=0.2414$, female $p=0.1805$ ) did not differ significantly among birds reared in different production systems. However, longer drumsticks in chickens of RB genotype (male $p=0.0165$, females $p=0.0245$ ) were observed. Females of RB genotype showed higher drumstick circumference $(p=0.0223)$ than other genotypes while males showed non-significant differences $(p=0.0394)$ in drumstick circumference among different genotypes.

Keel length of both the sexes showed non-significant differences (male $p=0.2588$, females $p=0.2001$ ) in all genotypes. Males reared in aviary systems showed higher $(p=0.0085)$ keel length than other males reared in enriched cage and conventional cage systems while females showed no influence $(p=0.7051)$ of production system on keel length. Non-significant differences in wing spread (male $p=0.7490$, females $p=0.2986$ ) and shank length (male $p=0.2950$, females $p=0.4024$ ) of different genotypes and wing spread (male $p=0.2473$, females $p=0.1291$ ) and shank length (male $p=0.7681$, females $p=0.7185$ ) of different production systems were observed. Non-significant interactions were found in the drumstick length (male $p=0.2424$, females $p=0.4785$ ), drumstick circumference (male $p=0.4542$, females $p=0.2838$ ), keel length (male $p=0.1484$, females $p=0.0859$ ), shank length (male $p=0.1820$, females $p=0.0360$ ) and wing spread (male $p=0.6199$ ). However, females showed significant differences $(p=0.0833)$ in wing spread among interaction groups. 


\section{Antibody response and serum chemistry}

Serum chemistry and antibody response of Naked Neck, Black Australorp and Rhode Island Red crossbreds were studied under alternative production systems (Table 6). Among different genotypes, highest glucose level $(p=0.0006)$ was observed in RR genotypes followed by $\mathrm{RB}, \mathrm{BR}$ and $\mathrm{BB}$ genotypes. Among different production systems, the highest glucose level $(p<0.0001)$ was observed in the chickens reared in aviary system followed by conventional cages and enriched cages. Antibody titer against ND $(p=0.0235)$ and $\mathrm{IB}(p=0.0004)$ were highest in the chickens reared in aviary systems followed by enriched cages and conventional cages. Significant interactions between genotypes and production systems were observed in glucose $(p<0.0001)$ and total proteins $(p=0.0176)$ among serum chemistry parameters. Similarly, significant interactions in antibody titer against IB ( $p=0.0073)$ was observed.

Table 6 - Serum chemistry of experimental birds.

\begin{tabular}{|c|c|c|c|c|c|c|c|c|c|c|}
\hline GT & PS & GLU & TP & ALB & GLB & UA & CRT & $\mathrm{CHO}$ & ND & $\mathrm{IB}$ \\
\hline RB & & $168.57 \pm 5.93^{a b}$ & $4.15 \pm 0.14$ & $2.78 \pm 0.08$ & $1.57 \pm 0.06$ & $6.87 \pm 0.45$ & $0.59 \pm 0.03$ & $141.15 \pm 2.66$ & $4.88 \pm 0.09$ & $3704.53 \pm 70.35$ \\
\hline $\mathrm{RR}$ & & $178.27 \pm 4.88^{a}$ & $4.60 \pm 0.13$ & $2.65 \pm 0.09$ & $1.59 \pm 0.06$ & $6.94 \pm 0.46$ & $0.58 \pm 0.05$ & $136.07 \pm 3.55$ & $4.61 \pm 0.08$ & $3660.36 \pm 69.61$ \\
\hline$B R$ & & $158.36 \pm 7.78^{b c}$ & $4.34 \pm 0.10$ & $2.66 \pm 0.07$ & $1.50 \pm 0.04$ & $6.79 \pm 0.52$ & $0.59 \pm 0.04$ & $138.84 \pm 3.24$ & $4.82 \pm 0.09$ & $3777.93 \pm 66.97$ \\
\hline BB & & $150.51 \pm 6.26^{c}$ & $4.41 \pm 0.16$ & $2.79 \pm 0.05$ & $1.59 \pm 0.05$ & $7.80 \pm 0.33$ & $0.51 \pm 0.01$ & $131.45 \pm 5.71$ & $4.77 \pm 0.08$ & $3640.27 \pm 54.34$ \\
\hline & CC & $163.89 \pm 5.44^{b}$ & $4.48 \pm 0.15$ & $2.69 \pm 0.07$ & $1.55 \pm 0.05$ & $7.21 \pm 0.31$ & $0.54 \pm 0.03$ & $137.30 \pm 2.62$ & $4.62 \pm 0.05^{b}$ & $3547.71 \pm 40.92^{c}$ \\
\hline & AV & $182.06 \pm 3.74^{a}$ & $4.42 \pm 0.09$ & $2.69 \pm 0.06$ & $1.58 \pm 0.04$ & $7.14 \pm 0.53$ & $0.55 \pm 0.03$ & $141.56 \pm 3.90$ & $4.93 \pm 0.09^{a}$ & $3845.61 \pm 52.06^{a}$ \\
\hline & EC & $145.83 \pm 4.66^{c}$ & $4.22 \pm 0.09$ & $2.78 \pm 0.06$ & $1.56 \pm 0.05$ & $6.95 \pm 0.30$ & $0.61 \pm 0.03$ & $131.77 \pm 3.44$ & $4.76 \pm 0.07^{\mathrm{ab}}$ & $3694.00 \pm 51.91^{b}$ \\
\hline RB & CC & $181.20 \pm 6.51^{a b}$ & $3.94 \pm 0.24^{c}$ & $2.71 \pm 0.21$ & $1.66 \pm 0.15$ & $7.40 \pm 0.58$ & $0.63 \pm 0.01$ & $132.87 \pm 2.73$ & $4.57 \pm 0.14$ & $3443.11 \pm 73.02^{d}$ \\
\hline RB & AV & $176.06 \pm 3.56^{\mathrm{ab}}$ & $4.34 \pm 0.20^{\mathrm{bc}}$ & $2.91 \pm 0.10$ & $1.55 \pm 0.10$ & $6.32 \pm 1.28$ & $0.56 \pm 0.07$ & $149.56 \pm 1.89$ & $5.10 \pm 0.04$ & $3865.49 \pm 93.90^{\mathrm{ab}}$ \\
\hline RB & EC & $148.45 \pm 11.17^{\mathrm{cd}}$ & $4.18 \pm 0.30^{\mathrm{bc}}$ & $2.72 \pm 0.13$ & $1.51 \pm 0.10$ & $6.88 \pm 0.27$ & $0.58 \pm 0.07$ & $141.02 \pm 4.51$ & $4.96 \pm 0.18$ & $3804.99 \pm 74.29^{a b c}$ \\
\hline RR & CC & $178.25 \pm 4.15^{\mathrm{ab}}$ & $5.13 \pm 0.18^{a}$ & $2.47 \pm 0.13$ & $1.58 \pm 0.11$ & $6.71 \pm 1.04$ & $0.58 \pm 0.12$ & $131.79 \pm 6.46$ & $4.58 \pm 0.09$ & $3674.49 \pm 70.27^{\mathrm{abcd}}$ \\
\hline RR & AV & $194.56 \pm 4.78^{a}$ & $4.39 \pm 0.12^{b c}$ & $2.57 \pm 0.14$ & $1.58 \pm 0.13$ & $7.42 \pm 0.86$ & $0.52 \pm 0.09$ & $145.61 \pm 4.10$ & $4.72 \pm 0.19$ & $3735.72 \pm 150.43^{\mathrm{abcd}}$ \\
\hline RR & $\mathrm{EC}$ & $162.01 \pm 6.75^{b c}$ & $4.29 \pm 0.02^{\mathrm{bc}}$ & $2.91 \pm 0.14$ & $1.60 \pm 0.11$ & $6.71 \pm 0.69$ & $0.62 \pm 0.07$ & $130.80 \pm 5.90$ & $4.53 \pm 0.17$ & $3570.86 \pm 145.30^{\text {bcd }}$ \\
\hline BR & CC & $160.81 \pm 8.45^{b c}$ & $4.53 \pm 0.20^{\mathrm{abc}}$ & $2.63 \pm 0.05$ & $1.37 \pm 0.06$ & $7.47 \pm 0.54$ & $0.45 \pm 0.03$ & $138.45 \pm 6.03$ & $4.60 \pm 0.14$ & $3558.52 \pm 98.00^{\mathrm{bcd}}$ \\
\hline $\mathrm{BR}$ & AV & $185.19 \pm 5.74^{\mathrm{a}}$ & $4.12 \pm 0.19$ & $2.63 \pm 0.14$ & $1.62 \pm 0.09$ & $6.79 \pm 1.48$ & $0.59 \pm 0.10$ & $141.17 \pm 8.14$ & $4.99 \pm 0.23$ & $3971.75 \pm 50.23^{a}$ \\
\hline $\mathrm{BR}$ & EC & $129.08 \pm 5.88^{d}$ & $4.37 \pm 0.11^{b c}$ & $2.73 \pm 0.20$ & $1.52 \pm 0.02$ & $6.10 \pm 0.44$ & $0.73 \pm 0.03$ & $136.91 \pm 3.19$ & $4.87 \pm 0.07$ & $3803.52 \pm 91.75^{\mathrm{abc}}$ \\
\hline BB & CC & $135.31 \pm 4.29^{d}$ & $4.32 \pm 0.35^{b c}$ & $2.95 \pm 0.06$ & $1.60 \pm 0.02$ & $7.27 \pm 0.37$ & $0.51 \pm 0.04$ & $146.10 \pm 3.06$ & $4.75 \pm 0.09$ & $3514.71 \pm 64.61^{\mathrm{cd}}$ \\
\hline BB & AV & $172.44 \pm 10.69^{a b}$ & $4.85 \pm 0.08^{\mathrm{ab}}$ & $2.66 \pm 0.08$ & $1.57 \pm 0.02$ & $8.04 \pm 0.71$ & $0.52 \pm 0.01$ & $129.90 \pm 12.07$ & $4.89 \pm 0.22$ & $3809.47 \pm 101.70^{a b c}$ \\
\hline BB & $\mathrm{EC}$ & $143.78 \pm 6.77^{\mathrm{cd}}$ & $4.05 \pm 0.24^{c}$ & $2.77 \pm 0.07$ & $1.61 \pm 0.17$ & $8.09 \pm 0.68$ & $0.50 \pm 0.03$ & $118.35 \pm 8.64$ & $4.68 \pm 0.07$ & $3596.65 \pm 50.30^{\mathrm{bcd}}$ \\
\hline SOV & & \multicolumn{9}{|c|}{ ANOVA } \\
\hline GT & & 0.0006 & 0.1496 & 0.4543 & 0.6651 & 0.3809 & 0.4790 & 0.3348 & 0.1694 & 0.3222 \\
\hline PS & & $<0.0001$ & 0.2796 & 0.5503 & 0.9211 & 0.8894 & 0.3545 & 0.1295 & 0.0235 & 0.0004 \\
\hline $\mathrm{GT} \times \mathrm{PS}$ & & $<0.0001$ & 0.0176 & 0.3388 & 0.8789 & 0.8392 & 0.4009 & 0.0667 & 0.1695 & 0.0073 \\
\hline
\end{tabular}

Note: Superscripts on different means within column differ significantly at $p \leq 0.05$.

$\mathrm{GT}=$ Genotype; RB = RNN $\times$ BNN; RR = RNN $\times$ RNN; BR = BNN $\times$ RNN; BR = BNN $\times$ RNN; BB $=B N N \times B N N ;$ PS $=$ Production System; CC $=$ Conventional Cages; $A V=$ Aviary; $\mathrm{EC}=$ Enriched Cages; SOV = Source of Variation; GLU = Glucose $(\mathrm{mg} / \mathrm{dL}) ; \mathrm{TP}=$ Total protein $(\mathrm{mg} / \mathrm{dL}) ; \mathrm{ALB}=\mathrm{Albumin}(\mathrm{mg} / \mathrm{dL}) ; \mathrm{GLB}=\mathrm{Globulin}(\mathrm{mg} / \mathrm{dL}) ; \mathrm{UA}=\mathrm{Uric}$ acid $(\mathrm{mg} / \mathrm{dL}) ; \mathrm{CRT}=$ Creatinine $(\mathrm{mg} / \mathrm{dL}) ; \mathrm{CHO}=$ Cholesterol $(\mathrm{mg} / \mathrm{dL}) ; \mathrm{ND}=$ Newcastle Disease (HI titer); IB = Infectious Bronchitis (ELISA titer); ANOVA = Analysis of Variance.

\section{Behavioral Parameters}

Significant differences were observed in behavioral pattern of males and females among different production systems (Table 7,8 ). As far as males are concerned, higher dust bathing $(p<0.0001)$ was observed in BB genotype than RR, RB and BR genotypes. The rest of the observing parameters including feeding ( $p=0.9315)$, jumping $(p=0.1032)$, perching $(p=0.9623)$, sitting $(p=0.0928)$ and walking $(p=0.3486)$ remained the same among the chickens of different genotypes. Among different production systems, higher dust bathing $(p<0.0001)$, feeding $(p<0.0001)$, jumping $(p<0.0001)$ and walking $(p<0.0001)$ were observed in the males reared in aviary system than in the males reared in other production systems. Perching behavior $(p<0.0001)$ was observed to be higher in the chickens reared in enriched cages than in the aviary system. sitting behavior $(p<0.0001)$ was highest in chickens reared in conventional cages followed by enriched cages and aviary system. Significant interactions among genotypes and production system were observed in the behavioral traits of males.

As far as behavioral parameters of females are concerned, non-significant differences were observed in the behavior of chickens from different genotypes. Among different production systems, higher dust bathing $(p<0.0001)$, feeding $(p<0.0001)$, jumping $(p<0.0001)$, and walking $(p<0.0001)$ were observed 
Table 7 - Behavioral response of male birds.

\begin{tabular}{|c|c|c|c|c|c|c|c|}
\hline GT & PS & Dust bathing & Feeding & Jumping & Perching & Sitting & Walking \\
\hline $\mathrm{RB}$ & & $13.69 \pm 0.84^{b}$ & $16.20 \pm 0.61$ & $0.78 \pm 0.14$ & $15.72 \pm 1.13$ & $14.75 \pm 2.20$ & $10.77 \pm 2.68$ \\
\hline $\mathrm{RR}$ & & $13.64 \pm 0.81^{b}$ & $16.05 \pm 0.63$ & $0.74 \pm 0.13$ & $15.82 \pm 1.13$ & $14.67 \pm 2.23$ & $10.69 \pm 2.67$ \\
\hline$B R$ & & $13.59 \pm 0.84^{b}$ & $16.20 \pm 0.61$ & $0.72 \pm 0.13$ & $15.72 \pm 1.13$ & $14.75 \pm 2.20$ & $10.77 \pm 2.68$ \\
\hline \multirow[t]{4}{*}{ BB } & & $14.22 \pm 0.85^{a}$ & $16.28 \pm 0.65$ & $0.73 \pm 0.13$ & $15.82 \pm 1.11$ & $14.31 \pm 2.30$ & $10.86 \pm 2.68$ \\
\hline & $C C$ & -- & $13.98 \pm 0.18^{c}$ & $0.03 \pm 0.00^{c}$ & -- & $23.66 \pm 0.09^{a}$ & $0.22 \pm 0.00^{c}$ \\
\hline & AV & $16.29 \pm 0.07^{a}$ & $19.13 \pm 0.27^{a}$ & $1.25 \pm 0.01^{\mathrm{a}}$ & $12.42 \pm 0.13^{b}$ & $3.36 \pm 0.12^{c}$ & $24.31 \pm 0.07^{a}$ \\
\hline & EC & $11.28 \pm 0.07^{b}$ & $15.45 \pm 0.16^{b}$ & $0.94 \pm 0.01^{b}$ & $19.12 \pm 0.12^{\mathrm{a}}$ & $16.77 \pm 0.12^{b}$ & $7.74 \pm 0.08^{b}$ \\
\hline RB & $C C$ & -- & $14.09 \pm 0.30^{c d}$ & $0.04 \pm 0.00^{d}$ & -- & $23.56 \pm 0.21^{a}$ & $0.21 \pm 0.00^{d}$ \\
\hline RB & AV & $16.21 \pm 0.05^{b}$ & $19.15 \pm 0.59^{a}$ & $1.29 \pm 0.04^{a}$ & $12.36 \pm 0.31^{b}$ & $3.71 \pm 0.12^{c}$ & $24.29 \pm 0.10^{a}$ \\
\hline RB & EC & $11.18 \pm 0.12^{d}$ & $15.38 \pm 0.29^{b c}$ & $1.01 \pm 0.04^{b}$ & $19.08 \pm 0.24^{a}$ & $16.98 \pm 0.22^{b}$ & $7.79 \pm 0.15^{b c}$ \\
\hline $\mathrm{RR}$ & CC & -- & $13.20 \pm 0.06^{d}$ & $0.03 \pm 0.00^{d}$ & -- & $23.58 \pm 0.05^{a}$ & $0.23 \pm 0.00^{d}$ \\
\hline RR & AV & $16.07 \pm 0.09^{b}$ & $18.98 \pm 0.13^{a}$ & $1.26 \pm 1.01^{a}$ & $12.45 \pm 0.29^{b}$ & $3.47 \pm 0.27^{c d}$ & $24.19 \pm 0.14^{a}$ \\
\hline RR & EC & $11.22 \pm 0.10^{d}$ & $15.97 \pm 0.09^{b}$ & $0.93 \pm 0.02^{b c}$ & $19.18 \pm 0.29^{a}$ & $16.97 \pm 0.09^{b}$ & $7.66 \pm 0.18^{b c}$ \\
\hline$B R$ & $C C$ & -- & $14.09 \pm 0.30^{\mathrm{cd}}$ & $0.03 \pm 0.00^{d}$ & -- & $23.79 \pm 0.26^{a}$ & $0.21 \pm 0.00^{d}$ \\
\hline BR & AV & $16.12 \pm 0.08^{b}$ & $19.15 \pm 0.59^{\mathrm{a}}$ & $1.22 \pm 0.05^{a}$ & $12.33 \pm 0.24^{b}$ & $3.35 \pm 0.34^{c d}$ & $24.43 \pm 0.13^{a}$ \\
\hline$B R$ & EC & $11.05 \pm 0.08^{d}$ & $15.38 \pm 0.29^{b c}$ & $0.92 \pm 0.02^{c}$ & $19.11 \pm 0.17^{a}$ & $16.82 \pm 0.25^{b}$ & $7.52 \pm 0.06^{c}$ \\
\hline BB & CC & -- & $14.53 \pm 0.46^{c d}$ & $0.03 \pm 0.00^{d}$ & -- & $23.71 \pm 0.16^{a}$ & $0.23 \pm 0.01^{d}$ \\
\hline BB & AV & $16.76 \pm 0.08^{a}$ & $19.23 \pm 0.84^{a}$ & $1.23 \pm 0.03^{\mathrm{a}}$ & $12.53 \pm 0.30^{b}$ & $2.91 \pm 0.13^{d}$ & $24.36 \pm 0.18^{a}$ \\
\hline BB & EC & $11.68 \pm 0.17^{c}$ & $15.08 \pm 0.46^{b c}$ & $0.92 \pm 0.01^{b c}$ & $19.12 \pm 0.31^{\mathrm{a}}$ & $16.33 \pm 0.30^{b}$ & $8.00 \pm 0.17^{b}$ \\
\hline SOV & & ANOVA & & & & & \\
\hline GT & & $<0.0001$ & 0.9315 & 0.1032 & 0.9623 & 0.0928 & 0.3486 \\
\hline PS & & $<0.0001$ & $<0.0001$ & $<0.0001$ & $<0.0001$ & $<0.0001$ & $<0.0001$ \\
\hline $\mathrm{GT} \times \mathrm{PS}$ & & $<0.0001$ & $<0.0001$ & $<0.0001$ & $<0.0001$ & $<0.0001$ & $<0.0001$ \\
\hline
\end{tabular}

Note: Superscripts on different means within column differ significantly at $p \leq 0.05$.

$\mathrm{GT}=$ Genotype; RB = RNN $\times$ BNN; RR = RNN $\times$ RNN; BR = BNN $\times$ RNN; BR = BNN $\times$ RNN; BB = BNN $\times$ BNN; PS = Production System; CC = Conventional Cages; $A V=$ Aviary; EC $=$ Enriched Cages; $\mathrm{SOV}=$ Source of Variation; $\mathrm{ANOVA}=$ Analysis of Variance.

Table 8 - Behavioral response of female birds.

\begin{tabular}{|c|c|c|c|c|c|c|c|}
\hline GT & PS & Dust bathing & Feeding & Jumping & Perching & Sitting & Walking \\
\hline $\mathrm{RB}$ & & $15.01 \pm 0.92$ & $14.65 \pm 0.47$ & $0.75 \pm 0.13$ & $17.89 \pm 1.00$ & $15.81 \pm 2.31$ & $9.89 \pm 2.37$ \\
\hline RR & & $15.23 \pm 0.85$ & $14.62 \pm 0.40$ & $0.74 \pm 0.13$ & $17.87 \pm 1.00$ & $15.74 \pm 2.32$ & $9.95 \pm 2.37$ \\
\hline BR & & $15.30 \pm 0.84$ & $14.63 \pm 0.42$ & $0.74 \pm 0.14$ & $17.83 \pm 0.97$ & $15.65 \pm 2.33$ & $9.87 \pm 2.42$ \\
\hline BB & & $15.15 \pm 0.92$ & $14.67 \pm 0.54$ & $0.74 \pm 0.13$ & $17.85 \pm 1.00$ & $15.69 \pm 2.32$ & $9.98 \pm 2.41$ \\
\hline & $C C$ & -- & $12.93 \pm 0.06^{c}$ & $0.04 \pm 0.00^{c}$ & -- & $25.08 \pm 0.08^{a}$ & $0.21 \pm 0.00^{c}$ \\
\hline & AV & $17.80 \pm 0.04^{a}$ & $16.57 \pm 0.28^{a}$ & $1.25 \pm 0.02^{\mathrm{a}}$ & $14.91 \pm 0.10^{b}$ & $4.14 \pm 0.06^{c}$ & $21.83 \pm 0.07^{a}$ \\
\hline & EC & $12.54 \pm 0.13^{b}$ & $14.43 \pm 0.20^{b}$ & $0.94 \pm 0.01^{b}$ & $20.81 \pm 0.14^{a}$ & $17.94 \pm 0.08^{b}$ & $7.72 \pm 0.07^{b}$ \\
\hline RB & CC & -- & $12.95 \pm 0.14^{c}$ & $0.04 \pm 0.00^{c}$ & -- & $25.10 \pm 0.19^{a}$ & $0.21 \pm 0.00^{d}$ \\
\hline RB & AV & $17.74 \pm 0.07^{a}$ & $16.61 \pm 0.66^{a}$ & $1.24 \pm 0.03^{a}$ & $14.99 \pm 0.38^{b}$ & $4.29 \pm 0.22^{c}$ & $21.64 \pm 0.09^{a}$ \\
\hline RB & EC & $12.28 \pm 0.34^{b}$ & $14.39 \pm 0.46^{b}$ & $0.97 \pm 0.02^{b}$ & $20.79 \pm 0.48^{a}$ & $18.03 \pm 0.22^{b}$ & $7.81 \pm 0.19^{b}$ \\
\hline RR & $C C$ & -- & $12.88 \pm 0.08^{c}$ & $0.03 \pm 0.00^{c}$ & -- & $25.15 \pm 0.15^{\mathrm{a}}$ & $0.23 \pm 0.00^{d}$ \\
\hline $\mathrm{RR}$ & AV & $17.77 \pm 0.05^{a}$ & $16.43 \pm 0.28^{a}$ & $1.24 \pm 0.03^{\mathrm{a}}$ & $14.85 \pm 0.04^{b}$ & $4.21 \pm 0.08^{c}$ & $21.72 \pm 0.21^{\mathrm{a}}$ \\
\hline $\mathrm{RR}$ & EC & $12.68 \pm 0.12^{b}$ & $14.54 \pm 0.11^{b}$ & $0.94 \pm 0.03^{b}$ & $20.88 \pm 0.14^{a}$ & $17.85 \pm 0.15^{b}$ & $7.91 \pm 0.10^{b}$ \\
\hline$B R$ & $C C$ & -- & $12.91 \pm 0.07^{c}$ & $0.04 \pm 0.00^{c}$ & -- & $25.10 \pm 0.22^{\mathrm{a}}$ & $0.20 \pm 0.01^{d}$ \\
\hline BR & AV & $17.81 \pm 0.12^{\mathrm{a}}$ & $16.52 \pm 0.43^{a}$ & $1.26 \pm 0.06^{a}$ & $14.96 \pm 0.22^{b}$ & $4.01 \pm 0.04^{c}$ & $21.98 \pm 0.16^{a}$ \\
\hline$B R$ & EC & $12.79 \pm 0.18^{b}$ & $14.46 \pm 0.26^{b}$ & $0.92 \pm 0.04^{b}$ & $20.70 \pm 0.30^{a}$ & $17.83 \pm 0.19^{b}$ & $7.43 \pm 0.12^{c}$ \\
\hline BB & $C C$ & -- & $12.98 \pm 0.22^{c}$ & $0.04 \pm 0.00^{c}$ & -- & $24.97 \pm 0.09^{a}$ & $0.21 \pm 0.01^{d}$ \\
\hline BB & AV & $17.89 \pm 0.05^{\mathrm{a}}$ & $16.70 \pm 0.90^{\mathrm{a}}$ & $1.25 \pm 0.02^{\mathrm{a}}$ & $14.84 \pm 0.12^{b}$ & $4.04 \pm 0.03^{c}$ & $21.99 \pm 0.08^{a}$ \\
\hline BB & EC & $12.41 \pm 0.35^{b}$ & $14.32 \pm 0.67^{b}$ & $0.92 \pm 0.02^{b}$ & $20.86 \pm 0.21^{\mathrm{a}}$ & $18.06 \pm 0.13^{b}$ & $7.73 \pm 0.12^{b c}$ \\
\hline SOV & & \multicolumn{6}{|c|}{ ANOVA } \\
\hline GT & & 0.5108 & 0.9990 & 0.9529 & 0.9966 & 0.6331 & 0.6859 \\
\hline PS & & $<0.0001$ & $<0.0001$ & $<0.0001$ & $<0.0001$ & $<0.0001$ & $<0.0001$ \\
\hline $\mathrm{GT} \times \mathrm{PS}$ & & $<0.0001$ & $<0.0001$ & $<0.0001$ & $<0.0001$ & $<0.0001$ & $<0.0001$ \\
\hline
\end{tabular}

Note: Superscripts on different means within column differ significantly at $p \leq 0.05$.

$\mathrm{GT}=$ Genotype; RB = RNN $\times$ BNN; RR = RNN $\times$ RNN; BR = BNN $\times$ RNN; BR = BNN $\times$ RNN; BB = BNN $\times$ BNN; PS = Production System; CC = Conventional Cages; $\mathrm{AV}=$ Aviary; EC $=$ Enriched Cages; $\mathrm{SOV}=$ Source of Variation; ANOVA $=$ Analysis of Variance. 
in the females reared in aviary system than in the females reared in other production systems. Perching $(p<0.0001)$ was observed to be higher in females reared in enriched cages than in the females reared in aviary system. Sitting $(p<0.0001)$ was highest in females reared in conventional cages followed by enriched cages and conventional cages. Significant interactions among genotypes and production system were observed in the behavioral traits of females.

\section{DISCUSSION}

\section{Morphometric measurements}

The present study revealed the effect of genotype and production systems on the growth, morphometric measurements, serum chemistry and antibody response. Body weight of both males and females were significantly higher in RB and BR genotypes when compared with RR and BB genotypes. This might be attributed to the additive gene action between genes of RNN and BNN genotypes. Similarly, both RB and BR genotypes showed significantly higher body length, shank length and drumstick lengths as compared to $B B$ and RR genotypes. This might be ascribed by the body weight differences in genotypes as the positive association between body weight and body measurements is the well-known fact. However, non-significant differences were observed among genotypes in terms of wing spread. The effect of crossbreeding between Rhode Island Red, Black Australorp and Naked neck were also explained by Ahmad et al. (2019a) where they reported the higher body weight, shank circumference and keel length of progeny obtained when Rhode Island Red and Black Australorp were crossed with naked neck chicken. Variations in morphological traits among genotypes has also been explained by Qureshi et al. (2018) where they reported the differences in varieties of Aseel chicken of Pakistan. Adekoya et al. (2013) reported dissimilarities in morphometric traits among different chicken genotypes of Nigeria. As far as the production system is concerned, higher body weight and body lengths were observed in enriched cage system when compared with aviary and conventional cage systems. Higher keel length of males was observed in aviary system whereas non-significant differences were observed in the drumstick length and drumstick circumference. The lowest body weight of chickens in the aviary system might be attributed to their vigorous activities, as birds have enough space to do more exercise thus utilizing more nutrients and burning of calories. Higher body lengths associated with higher body weights in enriched cage system might be due to less stress and more countenance of natural behavior. Availability of perches and dust bathing in enriched cages provides stress free compact space less than aviary to prevent nutrient wastage and more than conventional cage in which no materials were provided to express natural behavior. Higher shank circumference was observed in the conventional cage system which showed the negative association of shank circumference and perching behavior as conventional cages were not equipped with any perching material. Similar findings have also been observed by Ahmad et al. (2019a) who found lower body weight of chicken reared in free range system compared with semi intensive and intensive systems in which chickens were $9-14 \%$ heavier. The present findings are also in accordance with the observations of Olaniye et al. (2012) and Rehman et al. (2016). Lower body weight of slow growing broilers reared in free range system has also been explained by Stadig et al. (2016).

\section{Serum chemistry and antibody response}

In the present study, higher glucose level was observed in $B B$ genotype than in $R R, R B$ and $B R$ genotypes which might be attributed to the specific genetic makeup of BB chickens. Another possible reason behind the higher glucose level in $\mathrm{BB}$ genotype might be the insulin inhibition due to higher fat contents. However, in previous studies, non-significant effect of genotype on plasma glucose level has been reported (Ahmad et al., 2019a). In the present study, higher plasma glucose level was observed in chickens reared in aviary system followed by chickens reared in conventional cages and enriched cage. Similar findings have also been reported in previous studies in which significant variations has been observed in plasma glucose level of chickens reared in different production systems (Gunes et al., 2002, Rehman et al., 2016, Ahmad et al., 2019a). Non-significant variations in albumin, globulin, uric acid and blood cholesterol level was observed in the chickens of different production systems. Negligible effect of production systems on blood cholesterol level has been reported in previous studies (Elerogly et al., 2011, Diktas et al., 2015, Elerogly et al., 2015). As far as antibody response is concerned, the highest antibody titer against ND and IB was observed in chickens reared in aviary system followed by enriched cages and conventional cages. The highest antibody response in chickens reared in aviary system revealed its potential of acclimatization 
and survival in harsh climatic conditions. This might be attributed to stress free environment with better expression of natural behaviors in aviary system which may enhance the robustness against stressors and fighting ability against disease causing agents. This showed that increased stress in conventional cages inhibits the immune response against diseases while natural behaviors enhance the welfare traits and boosts immunity in aviary system. Ahmad et al. (2019a) also reported the higher antibody response in chickens reared in free range system when compared with those reared in semi intensive and intensive housing systems.

\section{Behavioral Parameters}

In the present study, higher dust bathing was observed in the males of BB genotype than in $R R, B R$ and RB genotypes which might be due to its specific genetic adaptation. As far as production systems are concerned, dust bathing, feeding, jumping and walking behavior was higher in the aviary system than in the enriched cages and conventional cages. This might be due to the availability of ample space which provoked explorative behavior and stimulated foraging. Chickens were provided with the opportunity to express natural doings which enhanced the comfort zone with reduced stress and encouraged activities. In previous studies, Ponte et al. (2008) observed increased foraging behavior of commercial broilers in free range area when compared with confined area. Similarly, increased feeding behavior of commercial layers was noticed when provided with free range access (Shimmura et al., 2008). Restricted explorative behaviors in confinement has been explained by Irfan et al. (2016) who reported higher immobility of turkeys (Maleagris gallopavo) in confinement than free range chickens. Mench et al. (2001) found that frequency of perching, leg stretching and wing flapping was increased when broilers were provided with outdoor access. Rehman et al. (2018) reported increased walking of indigenous Aseel chicken in part time free range. In the present study, sitting behavior was noticed to be highest in the conventional cages than in the enriched cages and aviary system which might be attributed to less moving capacity in conventional cages which dampens the activities and bird movements which were restricted to sitting and stimulated short duration expressions. Similar findings have been reported by Ahmad et al. (2019b) who found higher aggressive, standing and sitting behavior of chickens in intensive cage system. Rehman et al. (2018) reported higher standing and sitting behavior of Aseel chickens in confinement. Irfan et al. (2016) observed more frequent standing and sitting behavior of turkeys in intensive system than in free range system.

\section{CONCLUSIONS}

It can be concluded that RB and BR genotypes had better body weight and body length. Among genotypes, chickens reared in enriched cage system had better morphological traits while plasma glucose level and antibody response were more pronounced in aviary production system along with more explorative natural behavior.

\section{ACKNOWLEDGEMENTS}

The authors gratefully acknowledge the Administration at Indigenous Chicken Genetic Resource Centre, University of Veterinary and Animal Sciences, Ravi Campus, Pattoki, Pakistan for their help during the biological trial.

\section{CONFLICT OF INTEREST}

No potential conflict of interest was found by the authors.

\section{REFERENCES}

Adebambo AO, Ikeobi CON, Ozoje MO, Oduguwa OO, Adebambo OA Combining abilities of growth traits among pure and crossbred meat type chickens. Archivos de Zootecnia 2011;60:953-963.

Adekoya KO, Oboh BO, Adefenwa MA, Ogunkanmi LA. Morphological characterization of five Nigerian indigenous chicken types. Journal of Science Research and Development 2013;14:55-66.

Ahmad S, Mahmud A, Hussain J, Javed K. Morphometric traits, serum chemistry and antibody response of three chicken genotypes under free range, semi-intensive and intensive housing systems. Brazilian Journal of Poultry Science 2019a;21(1):1-8.

Ahmad S. Performance of rhode island red, black australorp and naked neck crossbred under free range, semi intensive and intensive housing systems [thesis]. Lahore (PK): University of Veterinary and Animal Sciences; 2019b.

Alikwe PCN, Faremi AY, Egwaikhide PA. Biochemical evaluation of serum metabolites, enzymes and haematological indices of broiler-chicks fed with varying levels of rumen epithelial scraps in place of fish meal proteins. Electronic Journal of Environmental, Agricultural and Food Chemistry 2010;9(6):991-999.

Baxter MR. The welfare problems of laying hens in battery cages. Veterinary Record 1994;134:614-619.

Costa LS, Pereira DF, Bueno LGF, Pandorfi H. Some aspects of chicken behaviour and welfare. Brazilian Journal of Poultry Science 2012;14(3):159-232. 
Craig JV, Swanson JC. Welfare perspectives on hens kept for egg production. Poultry Science 1994;73:921-938

Diktas M, Sekeroglu A, Duman M, Yildirim A. Effect of different housing systems on production and blood profile of slow-growing broilers. Kafkas Universitesi Veteriner Fakultesi Dergisi 2015;21:521-526.

Eleroglu H, Yalcin H, Yildirim A. Dietary effects of Ca-zeolite supplementation on some blood and tibial bone characteristics of broilers. South African Journal of Animal Science 2011;41:319-330.

Eleroglu H, Yıldırım A, Duman M, Sekeroglu A. The welfare of slow growing broiler genotypes reared in organic system. Emirates Journal of Food and Agriculture 2015;27:454-459.

Gunes N, Polat U, Petek M. Investigation of changes in biochemical parameters of hens raised in alternative housing systems. Uludag Universitesi Veteriner Fakultesi Dergisi 2002;21:39-42.

Haunshi S, Niranjan M, Shanmugam M, Padhi MK, Reddy MR, Sunitha $\mathrm{R}$, et al. Characterization of two Indian native chicken breeds for production, egg and semen quality, and welfare traits. Poultry Science 2011;90:314-320.

Irfan, Javid A, Ashraf M, Mahmud A, Altaf M, Hussain SM, et al. Timebudgets of turkeys (Maleagris gallopavo) reared under confinement and free range rearing systems. Pakistan Journal of Zoology 2016;48:19511956.

Kral I, Suchy P. Haematological studies in adolescent breeding cocks. Acta Veterinaria BRNO 2000;69:189-194.

Kumar B, Kumbhakar NK. Haemato-biochemical profile of Aseel in Chhattisgarh Region. Indian Veterinary Journal 2015;92:40-42.

Leeson S, Summers JD. Commercial poultry nutrition. $3^{\text {rd }}$ ed. Nottingham: Nottingham University Press; 2005. p.297-305.

Mallia JG. Observation on family poultry units in parts of Central America and sustainable development opportunities. Livestock Research and Rural Development 1999;11(3). Available from: https://www.Irrd.cipav. org.co//rrd11/3/mal113.htm

Mench JA, Garner JP, Falcone C. Behavioural activity and its effects on leg problems in broiler chickens. Proceedings of the $6^{\text {th }}$ European Symposium on Poultry Welfare; 2001; Kingston: Rural Industries Research and Development Corporation; 2001. p.152-156.
Menge EO, Kosgey IS, Kahi AK. Bio-economic model to support breeding of indigenous chicken in different production systems. International Journal of Poultry Science 2005;4:1-13.

Olaniyi OA, Oyenaiya OA, Sogunle OM, Akinola OS, Adeyemi OA, Ladokun $\mathrm{OA}$. Free-range and deep litter housing systems:effect on performance and blood profile of two strains of cockerel chickens. Tropical and Subtropical Agroecosystems 2012;15:511-523.

Ponte PIP, Rosado CMC, Crespo JP, Crespo DG. Pasture intake improves the performance and meat sensory attributes of free-range broilers. Poultry Science 2008;87:71-79.

Qureshi M, Qadri AH, Gachal GS. Morphological study of various varieties of Aseel chicken breed inhabiting district Hyderabad. Journal of Entomology and Zoology Study 2018;6(2):2043-2045.

Rehman MS, Mahmud A, Mehmood S, Pasha TN, Javed K, Hussain J, et al. Production performance of Aseel chicken under free range, semiintensive and confinement rearing systems. Journal of Animal and Plant Sciences 2016;26(6):1589-1596.

Rehman MS, Mahmud A, Mehmood S, Pasha TN, Khan MT, Hussain J. Assessing behavior in Aseel pullets under free-range, part-time free-range, and cage system during growing phase. Poultry Science 2018;97:725-732

Shimmura T, Suzuki T, Hirahara S, Eguchi Y, Uetake K, Tanaka T. Pecking behaviour of laying hens in single-tiered aviaries with and without outdoor area. British Poultry Science 2008;49:396-401.

Stadig LM, Rodenburg TB, Reubens B, Aerts J, Duquenne B, Tuyttens FAM. Effects of free-range access on production parameters and meat quality, composition and taste in slow-growing broiler chickens. Poultry Science 2016;95:2971-2978.

Tukey JW. The problem of multiple comparisons. In: Braun H. The collected works of John W. Tukey. Multiple comparisons. v.8. New York: Chapman and Hall; 1953.

Xie D, Wang ZX, Dong YL, Cao J, Wang JF, Chen JL, et al. Effects of monochromatic light on immune response of broilers. Poultry Science 2008;87:1535-1539. 